\title{
Complication rates following ventricular tachycardia ablation in ischaemic and non-ischaemic cardiomyopathies: a systematic review
}

\author{
Wern Yew Ding ${ }^{1,2}$ (D) Charles M. Pearman ${ }^{1,3} \cdot$ Laura Bonnett $^{4} \cdot$ Ahmed Adlan $^{1} \cdot$ Shui Hao Chin ${ }^{1} \cdot$ Nathan Denham ${ }^{3}$. \\ Simon Modi $^{1} \cdot$ Derick Todd $^{1} \cdot$ Mark C. S. Hall $^{1}$ - Saagar Mahida ${ }^{1,2}$
}

Received: 2 November 2020 / Accepted: 17 January 2021 / Published online: 29 January 2021

(C) The Author(s) 2021

\begin{abstract}
Background Catheter ablation of ventricular tachycardia (VT) is associated with potential major complications, including mortality. The risk of acute complications in patients with ischaemic cardiomyopathy (ICM) and non-ischaemic cardiomyopathy (NICM) has not been systematically evaluated.

Methods PubMed was searched for studies of catheter ablation of VT published between September 2009 and September 2019. Pre-specified primary outcomes were (1) rate of major acute complications, including death, and (2) mortality rate.

Results A total of 7395 references were evaluated for relevance. From this, 50 studies with a total of 3833 patients undergoing 4319 VT ablation procedures fulfilled the inclusion criteria (mean age 59 years; male 82\%; 2363 [62\%] ICM; 1470 [38\%] NICM). The overall major complication rate in ICM cohorts was 9.4\% (95\% CI, 8.1-10.7) and NICM cohorts was 7.1\% (95\% CI, 6.0-8.3). Reported complication rates were highly variable between studies (ICM I ${ }^{2}=90 \%$; NICM I ${ }^{2}=89 \%$ ). Vascular complications (ICM 2.5\% [95\% CI, 1.9-3.1]; NICM 1.2\% [95\% CI, 0.7-1.7]) and cerebrovascular events (ICM 0.5\% [95\% CI, 0.2-0.7]; NICM, $0.1 \%$ [95\% CI, 0-0.2]) were significantly higher in ICM cohorts. Acute mortality rates in the ICM and NICM cohorts were low (ICM 0.9\% [95\% CI, 0.5-1.3]; NICM 0.6\% [95\% CI, 0.3-1.0]) with the majority of overall deaths (ICM 75\%; NICM $80 \%$ ) due to either recurrent VT or cardiogenic shock.

Conclusion Overall acute complication rates of VT ablation are comparable between ICM and NICM patients. However, the pattern and predictors of complications vary depending on the underlying cardiomyopathy.
\end{abstract}

Keywords Catheter ablation - Ventricular tachycardia $\cdot$ Structural heart disease $\cdot$ Ischaemic cardiomyopathy $\cdot$ Non-ischaemic cardiomyopathy $\cdot$ Complications $\cdot$ Death $\cdot$ Mortality

\section{Introduction}

Ventricular tachycardia (VT) is a major cause of sudden death in patients with structural heart disease. While implantable cardiac defibrillators (ICD) reduce the risk of sudden death

Wern Yew Ding

dwyew@hotmail.com

1 Department of Cardiac Electrophysiology, Liverpool Heart and Chest Hospital, Liverpool, UK

2 Liverpool Centre for Cardiovascular Science, Liverpool, UK

3 Unit of Cardiac Physiology, Institute of Cardiovascular Sciences, Manchester Academic Health Sciences Centre, The University of Manchester, Manchester, UK

4 Department of Biostatistics, University of Liverpool, Liverpool, UK
$[1,2]$, recurrent ICD shocks due to VT remains an important cause of morbidity and mortality [3]. Catheter ablation is an effective technique for reducing the risk of recurrent VT and ICD therapies in patients with ischaemic and non-ischaemic cardiomyopathies (ICM; NICM) [4-6]. However, VT ablation is associated with a risk of major complications including mortality, and a decision to undertake VT ablation involves balancing these risks against potential benefits in terms of arrhythmia-free survival.

To date, complication rates associated with VT ablation have largely been defined based on data from single-centre studies or multicentre studies with limited sample sizes [7-9]. A comprehensive synthesis of available literature with a focus on procedure-related complications has not been undertaken. Furthermore, despite differing substrates, comorbidities and ablation strategies, the relative complication rates of VT ablation in patients with ICM and NICM are not 
fully defined. An in-depth understanding of complication rates has potential implications in terms of patient selection, ablation strategies and informed consent.

The aim of this systematic review was to assess the risk of major acute complications associated with catheter ablation of VT.

\section{Methods}

A literature search was performed using PubMed to identify all relevant studies published on catheter ablation of sustained VT in adults with structural heart disease between September 2009 and September 2019. This date range was selected in order to ensure that the findings are more applicable to current clinical practice. The study adhered to the Preferred Reporting Items for Systematic Reviews and Meta-analyses [10] and Meta-analysis of Observational Studies in Epidemiology guidelines for reporting [11]. Inclusion of other relevant studies not identified during our initial search was permitted. A description of our search strategy is summarised in Fig. 1.
Detailed search methodology is included in the Supplemental Material.

\subsection{Outcome measures}

The pre-specified primary outcome measures were the rates of (1) major acute complications, including death, and (2) mortality. To improve specificity and avoid including deaths unrelated to the procedure in these multimorbid cohorts, only deaths within the same admission or 7 days of VT ablation were included. The secondary outcome measure was the rate of specific complications. Acute complications included vascular access-related complications, pericardial effusion, cardiac perforation, need for cardiac surgery, complete atrioventricular block, cerebrovascular accident (CVA) or transient ischaemic attack (TIA), cardiogenic shock or severe pulmonary oedema, lead displacement, venous or arterial thromboembolism, myocardial infarction, other major bleeding, infection requiring antibiotics, phrenic nerve palsy and pneumothorax or haemothorax. Other major bleeding was based on studyspecific definitions that were not related to vascular access-
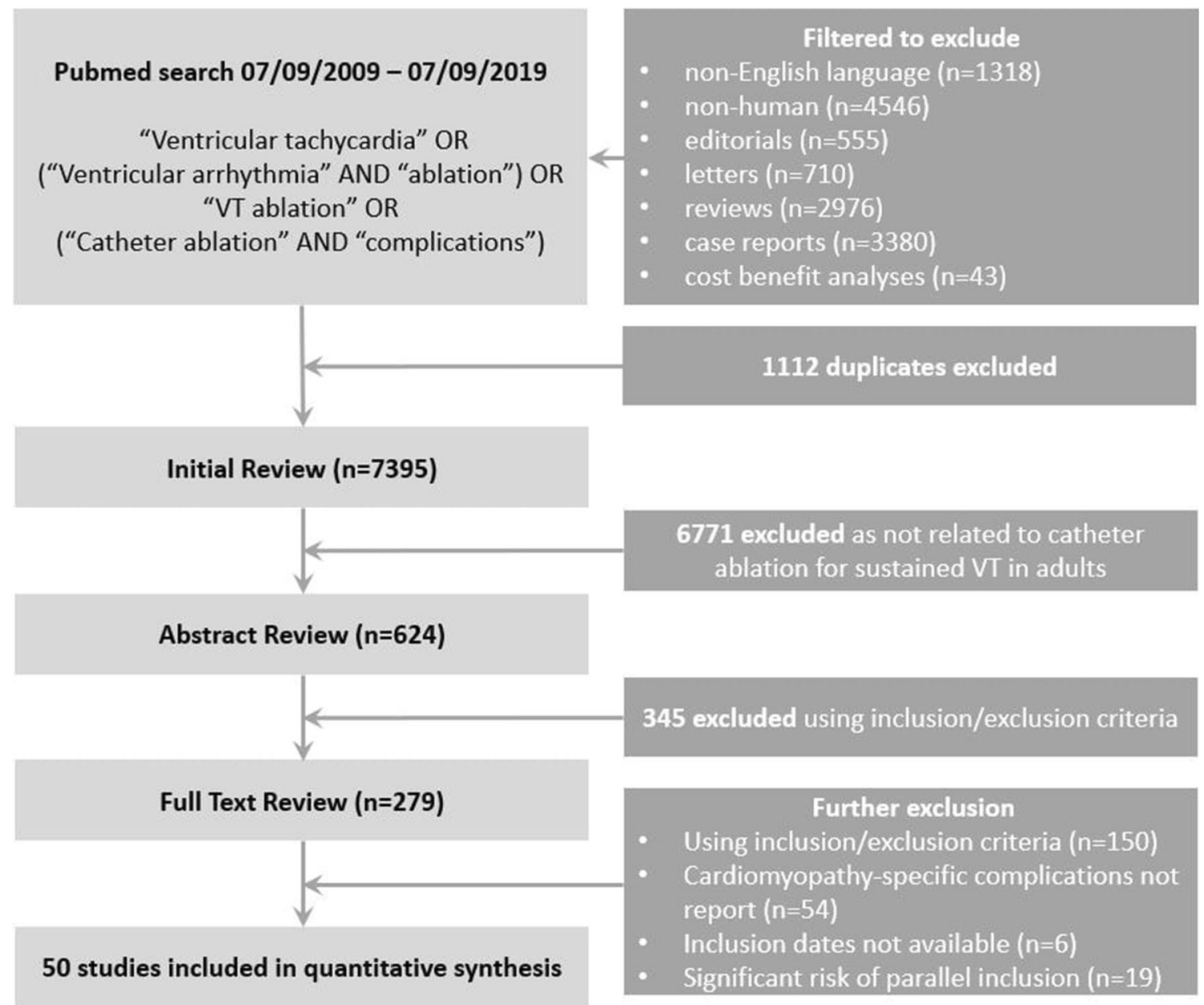

1112 duplicates excluded

6771 excluded as not related to catheter ablation for sustained VT in adults

\section{5 excluded using inclusion/exclusion criteria}

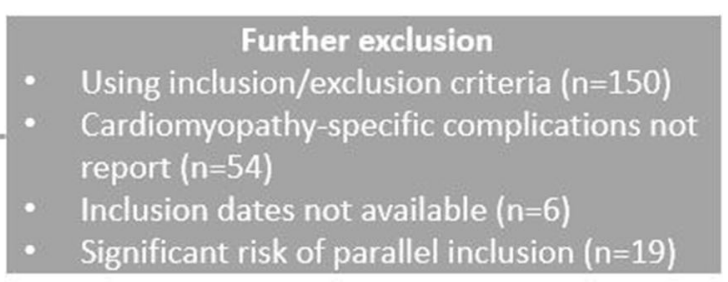

Fig. 1 Flow diagram of search strategy 
related complications, pericardial effusion or cardiac perforation.

\subsection{Study selection}

Inclusion criteria for studies were as follows: (1) participants aged $\geq 18$ years, (2) $\geq 95 \%$ study participants with VT ablation in the context of structural heart disease, (3) reporting of complications following VT ablation stratified by cardiomyopathy subtype and (4) $\geq 20$ patients undergoing VT ablation. Exclusion criteria for studies were as follows: (1) surgical ablation techniques were used; (2) $\geq 5 \%$ of participants who underwent catheter ablation had congenital heart disease; (3) $\geq 5 \%$ participants underwent catheter ablation of premature ventricular complexes alone; (4) $\geq 5 \%$ participants underwent catheter ablation for ventricular fibrillation; (5) animal models or in vitro studies; (6) not published in English; (7) abstracts, review articles, conference proceedings, editorials and metaanalyses; and (8) potential parallel inclusion of the same participants/cohorts from a single centre in multiple studies.

We applied a systematic approach to minimise parallel repetition. In situations where centres had reported patient cohorts in multiple studies, potential parallel inclusion of participants was identified based on the recruitment period. As such, studies with potential parallel inclusion in which the recruitment period was not specified were excluded. For the remainder, those with $<20 \%$ overlap of the recruitment period were included. Where multiple studies had $\geq 20 \%$ overlap of the recruitment period, only one study was included, prioritising randomised controlled trials (RCTs) over case-control and cohort studies. In the presence of multiple cohort studies only, the study with the largest cohort was prioritised.

\subsection{Data extraction and quality assessment}

All included manuscripts were assessed independently by two reviewers. Any differences were resolved by consensus. Quality assessment was performed using a list of 8-quality items that addressed both the internal and external validity of a study (Supplemental Material) [12].

\subsection{Statistical analysis}

Data analysis was performed using R (version 3.6) [13]. Pooled estimates were obtained across studies using a random effects model according to the DerSimonian and Laird method [14]. The $\mathrm{I}^{2}$ statistic was used to quantify heterogeneity between studies [15]. Univariable meta-regression was used to evaluate the impact of clinical covariates on major acute complications. A two-sided $p$ value of $<0.05$ was considered statistically significant. Risk of publication bias was assessed using Funnel plot and Egger's test. Study weighting was assigned using number of participants in each study rather than standard error to avoid excessive weighting of those reporting no complications. Studies with reported events rates of zero were assigned values of 0.005 to avoid dividing by a zero count which would yield a computational error.

\section{Results}

\subsection{Study characteristics}

The initial literature search yielded 7395 citations. After sequential filtering (7116 excluded following title/abstract review; 229 excluded after full text review), 50 studies were included in the final analysis ( 45 cohort studies, 4 randomised controlled trials and 1 case-control study). Forty-one (82\%) were single-centre studies. Overall, the 50 studies included 3833 patients who underwent a total of 4319 VT ablation procedures. Twenty-nine studies included an ICM cohort, while 23 studies included an NICM cohort. Histograms of the final recruitment period and publication dates are included in Fig. 2. Study characteristics are included in Supplemental Table 1.

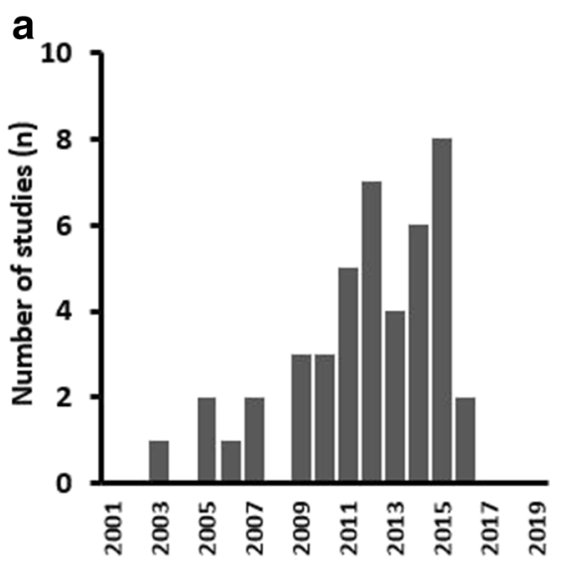

Recruitment completion (year)

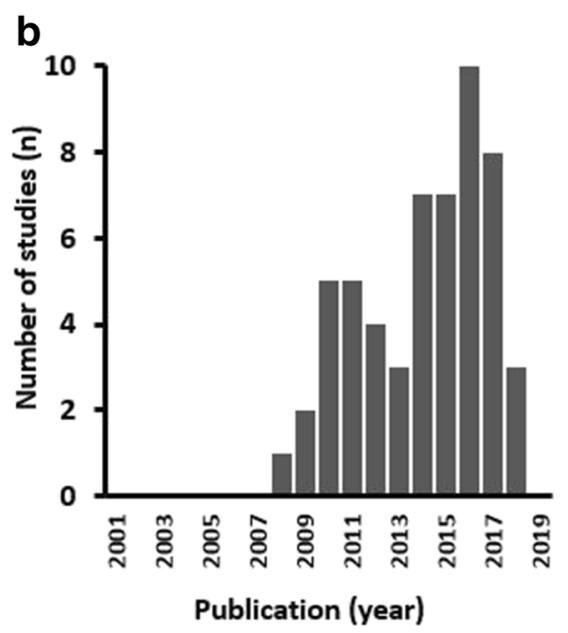

Fig. 2 Histograms for distribution of recruitment and publication dates 


\subsection{Demographics and procedural characteristics}

Overall, $82 \%$ of study subjects were male (mean age 59 years; mean left ventricular ejection function 37\%). Baseline demographics and procedural characteristics of the overall cohort are included in Supplemental Table 2. Data on ICD was available for $43(86 \%)$ studies. In total, $2973 / 3343$ (84.4\%) patients from these studies had an ICD either prior to or during the time of ablation. A total of 2363 (62\%) patients had ICM, while $1470(38 \%)$ had NICM. Compared to the NICM population, the mean age of the ICM population was higher (65 years vs 51 years) with a lower proportion of female patients (10\% vs $29 \%$ ) and re-do procedures (5\% vs $23 \%$ ). The procedure time was shorter for the ICM population (225 vs $273 \mathrm{~min}$ ) despite a longer ablation time (39 vs $32 \mathrm{~min}$ ). Eight percent of ablations in the ICM population involved combined endocardial/ epicardial access compared to $45 \%$ in the NICM population. ICM and NICM cohort-specific baseline and procedural characteristics are included in Table 1.

\subsection{Major complications}

Pooled major acute complications are included in Supplemental Table 3. The overall rate of major complication (including death) was $8.8 \%$ (95\% confidence interval (CI), 7.9-9.7). The most common complications were vascular access-related complications $(2.0 \%$ [95\% CI, 1.62.4]) and pericardial effusion (1.4\% [95\% CI, 1.1-1.8]). However, only a minority of these cases required intervention (vascular: $0.2 \%$ [95\% CI, $0.1-0.4]$; pericardial effusion: $0.9 \%$ [95\% CI, 0.6-1.1]). The major complication rate was comparable in ICM and NICM cohorts (ICM 9.4\% [95\% CI, 8.1-10.7] vs NICM 7.1\% [95\% CI, 6.08.3]). Overall significantly more vascular access-related complications and CVA/TIA were reported in ICM cohorts compared to NICM, $2.5 \%$ (95\% CI, 1.9-3.1) vs $1.2 \%(95 \%$ CI, 0.7-1.7) and $0.5 \%$ (95\% CI, 0.2-0.7) vs $0.1 \%$ (95\% CI, $0-0.2)$, respectively. Specific complications in ICM and NICM cohorts are included in Table 2.

Table 1 Demographics and procedural characteristics of ICM and NICM cohorts

\begin{tabular}{|c|c|c|c|c|c|c|c|c|c|c|}
\hline & \multicolumn{5}{|l|}{ ICM } & \multicolumn{5}{|l|}{ NICM } \\
\hline & $\begin{array}{l}\text { Number of } \\
\text { studies }\end{array}$ & Mean & SD & Minimum & Maximum & $\begin{array}{l}\text { Number of } \\
\text { studies }\end{array}$ & Mean & $\mathrm{SD}$ & Minimum & Maximum \\
\hline Males $(\%)$ & 27 & 90.1 & 6.2 & 71.4 & 100 & 23 & 71.5 & 15.0 & 30.6 & 90.9 \\
\hline Age (years) & 29 & 65.2 & 5.9 & 37.0 & 72.0 & 23 & 50.5 & 9.6 & 34.0 & 65.0 \\
\hline LV ejection fraction (\%) & 28 & 31.3 & 4.9 & 21.0 & 49.0 & 19 & 43.6 & 10.1 & 29.0 & 57.0 \\
\hline Diabetes $(\%)$ & 12 & 30.7 & 10.5 & 14.3 & 50.0 & 5 & 21.6 & 17.6 & 4.3 & 42.9 \\
\hline Renal failure (\%) & 6 & 13.8 & 8.0 & 0 & 22.6 & 3 & 21.2 & 12.8 & 8.9 & 34.5 \\
\hline VT storm (\%) & 17 & 51.6 & 35.2 & 0 & 100 & 13 & 50.3 & 30.3 & 17.8 & 100 \\
\hline NYHA class III or IV (\%) & 11 & 32.5 & 19.1 & 11.1 & 65.0 & 10 & 27.9 & 19.6 & 0 & 54.8 \\
\hline Prior catheter VT ablation (\%) & 18 & 4.5 & 9.7 & 0 & 29.6 & 16 & 23.0 & 27.8 & 0 & 100 \\
\hline Procedure time (minutes) & 23 & 225.2 & 81.2 & 104.0 & 453.0 & 16 & 273.3 & 110.0 & 161.0 & 480.0 \\
\hline Ablation time (minutes) & 15 & 39.2 & 31.2 & 9.0 & 114.0 & 11 & 32.2 & 17.0 & 11.0 & 70.0 \\
\hline \multicolumn{11}{|l|}{ Ablation site (\%) } \\
\hline LV only & 10 & 97.5 & 6.3 & 80.2 & 100 & 10 & 16.0 & 35.0 & 0 & 100 \\
\hline RV only & 10 & 0.1 & 0.4 & 0 & 1.4 & 10 & 83.1 & 34.7 & 0 & 100 \\
\hline Both LV and RV & 10 & 2.4 & 5.9 & 0 & 18.4 & 10 & 0.9 & 2.7 & 0 & 8.7 \\
\hline \multicolumn{11}{|l|}{ Access site $(\%)$} \\
\hline Endocardial only & 23 & 92.0 & 13.8 & 48.9 & 100 & 20 & 49.7 & 31.2 & 0 & 90.2 \\
\hline Epicardial only & 23 & 0.1 & 0.3 & 0 & 1.3 & 21 & 3.7 & 12.9 & 0 & 59.1 \\
\hline Combined endocardial/epicardial & 23 & 8.0 & 13.8 & 0 & 51.1 & 21 & 45.0 & 33.7 & 0 & 100 \\
\hline \multicolumn{11}{|l|}{ Approach type (\%) } \\
\hline Antegrade only & 9 & 44.0 & 45.1 & 0 & 100 & 4 & 3.9 & 4.6 & 0 & 8.7 \\
\hline Retrograde only & 9 & 40.3 & 44.1 & 0 & 97.7 & 3 & 52.1 & 50.1 & 0 & 100 \\
\hline Both ante- and retro-grade & 9 & 15.6 & 32.8 & 0 & 100 & 3 & 14.6 & 25.2 & 0 & 43.7 \\
\hline Pre-procedural anticoagulation (\%) & 14 & 100 & 0 & 100 & 100 & 4 & 90.0 & 20.0 & 60.0 & 100 \\
\hline
\end{tabular}

$I C M$ ischaemic cardiomyopathy, $L V$ left ventricular, NICM non-ischaemic cardiomyopathy, NYHA New York Heart Association, $R V$ right ventricular, $S D$ standard deviation, $V T$ ventricular tachycardia 
Table 2 Major acute complications in ICM and NICM cohorts

\begin{tabular}{|c|c|c|c|c|}
\hline & \multicolumn{2}{|l|}{$\operatorname{ICM}(n=29 *)$} & \multicolumn{2}{|l|}{$\operatorname{NICM}\left(n=23^{*}\right)$} \\
\hline & $\begin{array}{l}\% \text { Pooled } \\
\text { complication rate } \\
(95 \% \mathrm{CI})\end{array}$ & $\begin{array}{l}\mathrm{I}^{2} \\
\text { statistic } \\
(\%)\end{array}$ & $\begin{array}{l}\% \text { Pooled } \\
\text { complication rate } \\
(95 \% \mathrm{CI})\end{array}$ & $\begin{array}{l}\mathrm{I}^{2} \\
\text { statistic } \\
(\%)\end{array}$ \\
\hline Any major acute complications & $9.40(8.07-10.73)^{+}$ & 90 & $7.14(5.95-8.33)^{\S}$ & 89 \\
\hline Death & $0.92(0.51-1.33)^{+}$ & 0 & $0.63(0.26-1.00)^{\S}$ & 0 \\
\hline $\begin{array}{l}\text { Any vascular access-related com- } \\
\text { plications }\end{array}$ & $2.53(1.92-3.14)$ & 60 & $1.16(0.67-1.65)$ & 8 \\
\hline $\begin{array}{l}\text { Vascular access-related } \\
\text { complications requiring } \\
\text { intervention }\end{array}$ & $0.21(0.03-0.39)$ & 0 & $0.23(0.01-0.44)$ & 0 \\
\hline Any pericardial effusion & $1.58(1.08-2.07)$ & 31 & $1.27(0.76-1.78)$ & 15 \\
\hline $\begin{array}{l}\text { Pericardial effusion requiring } \\
\text { drainage }\end{array}$ & $0.67(0.35-0.99)$ & 0 & $1.16(0.67-1.65)$ & 6 \\
\hline Need for cardiac surgery & $0.25(0.05-0.45)$ & 0 & $0.39(0.10-0.68)$ & 0 \\
\hline Complete AV block & $0.54(0.25-0.83)$ & 0 & $0.17(0-0.36)$ & 0 \\
\hline CVA or TIA & $0.46(0.19-0.73)$ & 0 & $0.06(0-0.17)$ & 0 \\
\hline Cardiac perforation & $0.17(0.01-0.34)$ & 0 & $0.28(0.04-0.52)$ & 0 \\
\hline $\begin{array}{l}\text { Cardiogenic shock or severe } \\
\text { pulmonary oedema }\end{array}$ & $0.42(0.16-0.67)$ & 0 & $0.12(0-0.27)$ & 0 \\
\hline Lead displacement & $0.38(0.14-0.62)$ & 0 & $0.01(0-0.04)$ & 0 \\
\hline $\begin{array}{l}\text { Venous or arterial } \\
\text { thromboembolism }\end{array}$ & $0.05(0-0.13)$ & 0 & $0.44(0.14-0.75)$ & 0 \\
\hline MI & $0.09(0-0.21)$ & 0 & $0.23(0.01-0.44)$ & 0 \\
\hline Other major bleeding & $0.13(0-0.27)$ & 0 & $0.06(0-0.17)$ & 0 \\
\hline Infection requiring antibiotics & $0.09(0-0.21)$ & 0 & $0.01(0-0.04)$ & 0 \\
\hline Phrenic nerve palsy & $0.05(0-0.13)$ & 0 & $0.12(0-0.27)$ & 0 \\
\hline Pneumothorax or haemothorax & $0.13(0-0.27)$ & 0 & $0.01(0-0.04)$ & 0 \\
\hline $\begin{array}{l}{ }^{*} \text { Unless otherwise stated } \\
{ }^{+} n=24 \\
{ }^{\S} n=19\end{array}$ & & & & \\
\hline
\end{tabular}

\subsection{Acute mortality}

The acute mortality rate (mortality rate during the same admission or within 7 days of VT ablation) was $0.9 \%$ (95\% CI, $0.6-1.1)$. Mortality rates in the ICM and NICM cohorts were comparable (ICM $0.9 \%$ [95\% CI, 0.5-1.3] vs NICM $0.6 \%$ [95\% CI, 0.3-1.0]). The mode of death was available in 20 of the 21 reported ICM deaths (9 [45\%] recurrence of ventricular arrhythmia, 6 [30\%] cardiogenic shock, 1 [5\%] hepatorenal failure, 1 [5\%] septic shock, 1 [5\%] pneumonia, 1 [5\%] acute respiratory distress syndrome, 1 [5\%] left ventricular perforation). The mode of death was available in 10 of 12 of the reported NICM deaths (7 [70\%] cardiogenic shock, 1 $[10 \%]$ recurrence of ventricular arrhythmia, 1 [10\%] left ventricular perforation, 1 [10\%] pulmonary embolism). A forest plot of the major complications is included in Fig. 3.

\subsection{Heterogeneity}

We observed significant heterogeneity between studies in terms of reported overall complication rates $\left(\mathrm{I}^{2}=90 \%\right.$, Fig. $3)$. When considering ICM and NICM separately, the degree of heterogeneity in complication rates remained high for each subtype $\left(\mathrm{ICM} \mathrm{I}^{2}=90\right.$; NICM I $\left.{ }^{2}=89\right)$. Of note, when considering RCTs only, we also observed a moderate to high degree of heterogeneity for overall complications $\left(\mathrm{I}^{2}=45 \%\right)$ and common complications (vascular access-related complications $\left[\mathrm{I}^{2}=24 \%\right]$, pericardial effusion $\left[\mathrm{I}^{2}=59 \%\right]$ and lead displacement $\left.\left[I^{2}=60 \%\right]\right)$. Similarly, there was a high degree of heterogeneity for overall complications $\left(\mathrm{I}^{2}=91 \%\right)$ when we limited our analysis to studies with $>100$ patients. We did not observe significant heterogeneity between studies $\left(I^{2}=0 \%\right)$ when considering acute mortality alone. 


\begin{tabular}{|c|c|c|}
\hline Study & $\%[95 \% \mathrm{Cl}]$ & Weighting \\
\hline Tanner (2009) & $9.5[2.3,16.8]$ & $1.6 \%$ \\
\hline Kuck (2010) & $11.5[2.9,20.2]$ & $1.3 \%$ \\
\hline Pluta(2010) & $0[0,0.7]$ & $0.5 \%$ \\
\hline Kuhne (2010) & $8.6[0,17.8]$ & $0.9 \%$ \\
\hline Deneke (2010) & $3.5[0.1,6.8]$ & $2.9 \%$ \\
\hline Santangeli (2010) & $0[0,0.7]$ & $0.6 \%$ \\
\hline Silva (2011) & $7.7[0,17.9]$ & $0.7 \%$ \\
\hline Alzand (2011) & $9.7[0,20.1]$ & $0.8 \%$ \\
\hline Bai (2011) & $6.1[0,12.8]$ & $1.3 \%$ \\
\hline Kozluk (2011) & $0[0,0.5]$ & $0.7 \%$ \\
\hline Dello Russo (2012) & $0[0,0.7]$ & $0.5 \%$ \\
\hline Philips (2012) & $1.7[0,3.6]$ & $4.5 \%$ \\
\hline Di Biase (2012) & $2.2[0,5.2]$ & $2.4 \%$ \\
\hline Arenal (2012) & $0[0,0.2]$ & $1.5 \%$ \\
\hline Pauriah (2013) & $8.9[0.6,17.2]$ & $1.2 \%$ \\
\hline Piers (2013) & $8.9[0.6,17.2]$ & $1.2 \%$ \\
\hline $\operatorname{Dinov}(2013)$ & $23.8[18.2,29.3]$ & $5.8 \%$ \\
\hline Mork (2014) & $10.7[5.2,16.3]$ & $3.1 \%$ \\
\hline Chopra (2014) & $0[0,0.7]$ & $0.5 \%$ \\
\hline Oloriz (2014) & $16.1[8.4,23.8]$ & $2.2 \%$ \\
\hline Silberbauer (2014) & $15.6[10.0,21.3]$ & $4.1 \%$ \\
\hline Proietti (2014) & $38.7[30.7,46.7]$ & $3.6 \%$ \\
\hline Dinov (2015) & $3.6[0,8.6]$ & $1.4 \%$ \\
\hline $\operatorname{Jin}(2015)$ & $5.0[0,11.8]$ & $1.0 \%$ \\
\hline Luther (2015) & $0[0,0.6]$ & $0.6 \%$ \\
\hline Santangeli (2015) & $5.0[1.1,8.8]$ & $3.1 \%$ \\
\hline Muser (2016) & $4.3[2.4,6.2]$ & $11.3 \%$ \\
\hline Souissi(2016) & $5.4[0.8,10.1]$ & $2.4 \%$ \\
\hline Di Biase (2016) & $5.1[1.1,9.0]$ & $3.0 \%$ \\
\hline Skoda (2016) & $0[0,0.3]$ & $1.4 \%$ \\
\hline Marchlinski (2016) & $5.2[2.3,8.0]$ & $6.0 \%$ \\
\hline Mussigbrodt (2016) & $6.5[1.5,11.6]$ & $2.4 \%$ \\
\hline $\operatorname{Lin}(2016)$ & $0[0,0.2]$ & $1.8 \%$ \\
\hline Muser (2016) & $6.8[0,14.3]$ & $1.1 \%$ \\
\hline Kuck (2017) & $13.0[4.0,21.9]$ & $1.4 \%$ \\
\hline Guo (2017) & $33.3[17.2,49.4]$ & $0.8 \%$ \\
\hline Kuroki (2017) & $2.8[0,5.8]$ & $2.8 \%$ \\
\hline $\operatorname{Jin}(2017)$ & $0[0,0.3]$ & $1.4 \%$ \\
\hline Nayyar (2017) & $0[0,0.5]$ & $0.7 \%$ \\
\hline Wei (2017) & $0[0,0.2]$ & $1.8 \%$ \\
\hline Kirubakaran (2017) & $0[0,0.5]$ & $0.7 \%$ \\
\hline Pappone (2017) & $0[0,0.1]$ & $3.5 \%$ \\
\hline Siontis (2018) & $20.7[15.3,26.1]$ & $5.6 \%$ \\
\hline Wolf (2018) & $10.7[5.9,15.5]$ & $4.1 \%$ \\
\hline Pooled estimate & $8.8[7.9,9.7]$ & \\
\hline
\end{tabular}

$8.8[7.9,9.7]$

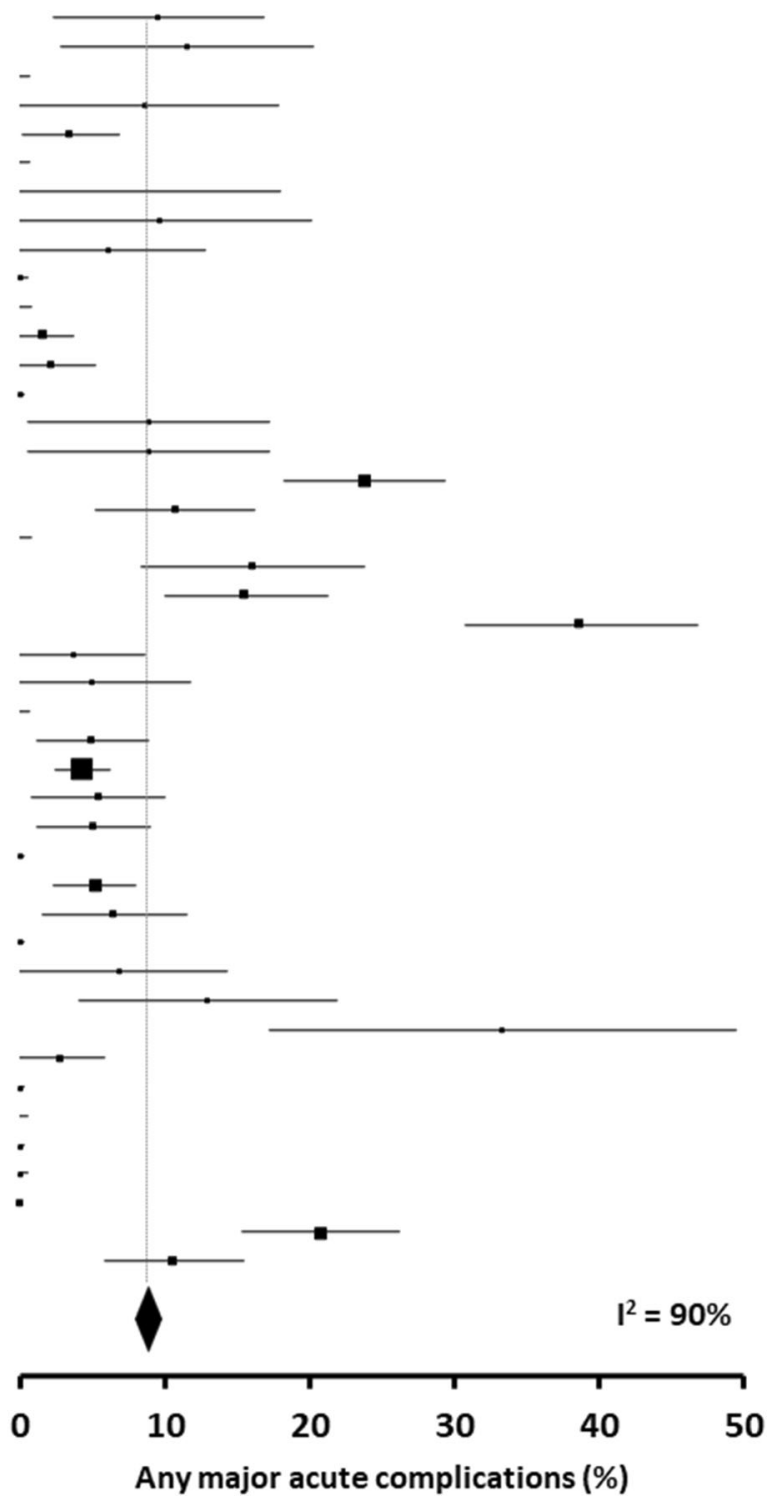

Fig. 3 Forest plot of acute major complications from each study

\subsection{Temporal trends of complication rates}

In order to determine whether there was a temporal trend in complication rates, we compared studies reported in the first 5 years of our inclusion period (2009-2014) to studies reported in the second 5 years (2015-2019). Despite broadly comparable baseline and procedural characteristics, no significant difference in major acute complications was seen between these two time periods in either ICM or NICM cohorts (ICM: 2009$2014,9.7 \%$ [95\% CI, 7.5-11.8] vs 2014-2019, 9.2\% [95\% CI, 7.4-11.0]; NICM: 2009-2014, 6.3\% [95\% CI, 3.8-8.9] vs 2014-2019, 7.4\% [95\% CI, 6.1-8.8]) (Supplemental Tables 4-7).

\subsection{Study quality and risk of bias}

The quality of studies included was variable using the predefined quality assessment criteria (Supplemental Materials): $5(10 \%)$ met all eight criteria; 27 (54\%) met at least six criteria; and $11(22 \%)$ met four or less criteria. A detailed description of the study population was provided in 42 studies (84\%), and complication rates were prospectively collected in 24 studies (48\%). Funnel plot and Egger's test were used to assess the risk of publication bias (Fig. 4). The Funnel plot was drawn using $n$ on the vertical axis as the more commonly used standard error was unduly influenced by small studies report zero complication rates [16]. While Egger's test was significant 


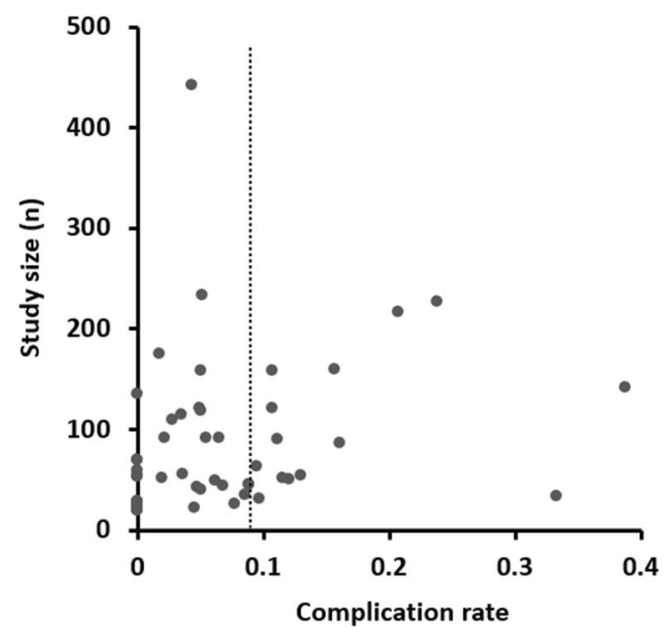

Fig. 4 Funnel plot of studies

$(z=13.2103, p<0.0001)$ suggesting possible publication bias, the plot was symmetrical on visual assessment. Furthermore, it is likely that confounding factors such as case mix and experience may have influenced the relationship between study size and reported complication rate separate to publication bias.

\subsection{Sensitivity analyses}

In keeping with our primary analysis, when only prospective studies ( $n=24 ; 2135$ patients) and RCTs were included ( $n=$ 4; 356 patients), the rates of major complications were $6.5 \%$ (95\% CI, 4.1-10.2) and 8.5\% (95\% CI, 3.1-13.9), respectively. The results are included in Supplemental Tables 8 and 9.

\section{Discussion}

The main findings from this systematic analysis which included 4319 VT ablation procedures are as follows: (1) the risk of mortality in the acute phase post-VT ablation is low $(<1 \%)$ in both ICM and NICM cohorts, with approximately three quarters of the reported cases attributed to recurrent ventricular arrhythmias or cardiogenic shock; (2) the overall major acute complications rates following VT ablation were comparable in ICM and NICM cohorts; (3) the profile of complications differs between ICM and NICM, with vascular access-related complications and CVA/TIA occurring with a significantly higher frequency in ICM cohorts; and (4) there was a high degree of variability in complication rates between studies.

The mortality rate in the acute phase post-procedure was low $(<1 \%)$, with the predominant causes of death being recurrent ventricular arrhythmias and cardiogenic shock (75-80\%). Amongst those patients with recurrent ventricular arrhythmias, mortality is attributable to a failure to alter the trajectory of the disease process rather than a direct procedure-related complication. In the ICM cohort, recurrence of VT accounted for close to half of the mortality cases. Understanding the relationship between VT ablation and cardiogenic shock is more complex. On the one hand, cardiogenic shock may represent the endpoint of the natural trajectory of the disease, while on the other, haemodynamic instability during an ablation procedure may have an adverse effect on cardiac pump function. Distinguishing between procedure-related complications and disease trajectory has important implications in terms of patient consent.

The variability in reported complication rates is a potentially important finding of this study. The complication rates ranged from 0 to $38.7 \%$. Given the variability in study quality, a proportion of the observed heterogeneity is likely to be attributable to differences in complication reporting and the influence of smaller studies with zero complication rates. However, a significant proportion in the variability is likely to be accounted by true variation in complication rates. This point is underscored by the fact that we observed moderate to high degree of heterogeneity even when considering prospective studies and RCTs alone. There are a number of potential explanations for variability. Firstly, we observed significant variation in patient baseline characteristics between studies. Secondly, procedural characteristics including access route and ablation time varied significantly between studies. Thirdly, there is potential variation in the level of expertise between different centres. Fourthly, the timing of VT ablation is likely to vary between centres. Finally, ablation strategies were highly variable between studies. For instance, VT ablation techniques guided by advanced image-integration techniques, more refined electrogram detection and ablation strategies that avoid VT induction are predicted to reduce procedure-related risk [17]. Overall, complication rates are likely to vary significantly due to a complex interaction between patient-specific and operator-specific factors. Our findings highlight the point that reliance on individual-centre or individual-study data to define complication rates is related with important limitations.

While the overall risk of complications was comparable between ICM and NICM cohorts, as discussed above, we observed a high degree of variability in complication rates between studies. Therefore, the comparisons between ICM and NICM in terms of absolute complication rates should be interpreted with caution. Our results do however indicate that the profile of complications between ICM and NICM is different. Vascular access-related complications and CVA/TIA were twofold and eightfold higher amongst ICM patients, respectively. Of note, however, only a small minority of patients required vascular interventions. This observation is in keeping with a higher prevalence of atherosclerotic vascular disease in ICM patients. Our findings underscore the importance of careful selection of the access route in ICM patients undergoing VT ablation. 
Overall, the findings of present study indicate that the mortality associated with VT ablation is low. Furthermore, the need for further interventions due to complications remains low. The study also highlights the challenges associated with defining complication rates following VT ablation. Generalisation of results from individual studies is associated with potential challenges, and individualised risk stratification strategies are needed. Our findings highlight the need for potential patient-level meta-analyses in the future to better understand complication rates in different subsets of patients undergoing VT ablation.

\section{Limitations}

One of the inherent limitations of this systematic analysis which is inclusive of a range of studies is a risk of publication, selection and reporting bias. We attempted to minimise these effects by employing a rigorous search strategy, using a strict inclusion and exclusion criteria and excluding studies with very small cohorts $(n<20)$. Furthermore, there was a significant variability in the quality scores of studies. The active decision to maintain inclusion of studies with low quality scores was supported by Stein et al. that found no relationship between these scores and study outcomes [18]. Patient selection, the timing of VT ablation, the specific ablation strategy and evolving technologies have an important impact on potential complication rates. Due to a lack of detailed data, we were unable to define the impact of these variables on complication rates. It is important to note that this study did not involve direct comparisons between ICM and NICM cohorts; therefore, conclusions regarding relative complication rates should be drawn with caution. Finally, the results should be interpreted in the context of the studies cited, and it is worth highlighting that VT ablation is a procedure where operator skill and patient selection may be limited.

Supplementary Information The online version contains supplementary material available at https://doi.org/10.1007/s10840-021-00948-6.

Data availability The data that support the findings of this study are available from the corresponding author, WYD, upon reasonable request.

\section{Compliance with ethical standards}

Conflict of interest The author declare no competing interests.

Code availability Not applicable.
Open Access This article is licensed under a Creative Commons Attribution 4.0 International License, which permits use, sharing, adaptation, distribution and reproduction in any medium or format, as long as you give appropriate credit to the original author(s) and the source, provide a link to the Creative Commons licence, and indicate if changes were made. The images or other third party material in this article are included in the article's Creative Commons licence, unless indicated otherwise in a credit line to the material. If material is not included in the article's Creative Commons licence and your intended use is not permitted by statutory regulation or exceeds the permitted use, you will need to obtain permission directly from the copyright holder. To view a copy of this licence, visit http://creativecommons.org/licenses/by/4.0/.

\section{References}

1. Moss AJ, Zareba W, Hall WJ, Klein H, Wilber DJ, Cannom DS, et al. Prophylactic implantation of a defibrillator in patients with myocardial infarction and reduced ejection fraction. N Engl J Med. 2002;346(12):877-83. https://doi.org/10.1056/NEJMoa013474.

2. Bardy GH, Lee KL, Mark DB, Poole JE, Packer DL, Boineau R, et al. Amiodarone or an implantable cardioverter-defibrillator for congestive heart failure. N Engl J Med. 2005;352(3):225-37. https://doi.org/10.1056/NEJMoa043399.

3. Proietti R, Labos C, Davis M, Thanassoulis G, Santangeli P, Russo $\mathrm{V}$, et al. A systematic review and meta-analysis of the association between implantable cardioverter-defibrillator shocks and longterm mortality. Can J Cardiol. 2015;31(3):270-7. https://doi.org/ 10.1016/j.cjca.2014.11.023.

4. Fernandez-Armenta J, Andreu D, Penela D, Trucco E, Cipolletta L, Arbelo E, et al. Sinus rhythm detection of conducting channels and ventricular tachycardia isthmus in arrhythmogenic right ventricular cardiomyopathy. Heart Rhythm. 2014;11(5):747-54. https://doi. org/10.1016/j.hrthm.2014.02.016.

5. Kuck K-H, Schaumann A, Eckardt L, Willems S, Ventura R, Delacretaz E, et al. Catheter ablation of stable ventricular tachycardia before defibrillator implantation in patients with coronary heart disease (VTACH): a multicentre randomised controlled trial. Lancet. 2010;375(9708):31-40. https://doi.org/10.1016/S01406736(09)61755-4.

6. Sapp JL, Wells GA, Parkash R, Stevenson WG, Blier L, Sarrazin J$\mathrm{F}$, et al. Ventricular tachycardia ablation versus escalation of antiarrhythmic drugs. N Engl J Med. 2016;375(2):111-21. https://doi. org/10.1056/NEJMoa1513614.

7. Barra S, Begley D, Heck P, Turner I, Agarwal S. Ablation of ventricular tachycardia in the very elderly patient with cardiomyopathy: how old is too old? Can J Cardiol. 2015;31(6):717-22. https://doi. org/10.1016/j.cjca.2014.12.016.

8. Mahapatra S, Tucker-Schwartz J, Wiggins D, Gillies GT, Mason $\mathrm{PK}$, McDaniel G, et al. Pressure frequency characteristics of the pericardial space and thorax during subxiphoid access for epicardial ventricular tachycardia ablation. Heart Rhythm. 2010;7(5):604-9. https://doi.org/10.1016/j.hrthm.2010.01.011.

9. Bunch TJ, Darby A, May HT, Ragosta M, Lim DS, Taylor AM, et al. Efficacy and safety of ventricular tachycardia ablation with mechanical circulatory support compared with substrate-based 
ablation techniques. Europace. 2012;14(5):709-14. https://doi.org/ 10.1093/europace/eur347.

10. Moher D, Liberati A, Tetzlaff J, Altman DG. Preferred reporting items for systematic reviews and meta-analyses: the PRISMA statement. PLoS Med. 2009;6(7):e1000097. https://doi.org/10.1371/ journal.pmed.1000097.

11. Stroup DF, Berlin JA, Morton SC, Olkin I, Williamson GD, Rennie D, et al. Meta-analysis of observational studies in epidemiology: a proposal for reporting. Meta-analysis of Observational Studies in Epidemiology (MOOSE) group. JAMA. 2000;283(15):2008-12.

12. Gupta A, Perera T, Ganesan A, Sullivan T, Lau DH, RobertsThomson KC, et al. Complications of catheter ablation of atrial fibrillation: a systematic review. Circ Arrhythm Electrophysiol. 2013;6(6):1082-8. https://doi.org/10.1161/CIRCEP.113.000768.

13. Core Team R. R: a language and environment for statistical computing. Vienna: R Foundation for Statistical Computing; 2019. Retrieved from https://www.r-project.org

14. DerSimonian R, Laird N. Meta-analysis in clinical trials. Control Clin Trials. 1986;7(3):177-88.
15. Higgins JPT, Thompson SG. Quantifying heterogeneity in a metaanalysis. Stat Med. 2002;21(11):1539-58. https://doi.org/10.1002/ sim.1186.

16. Sterne JA, Egger M. Funnel plots for detecting bias in meta-analysis: guidelines on choice of axis. J Clin Epidemiol. 2001;54(10): 1046-55. https://doi.org/10.1016/s0895-4356(01)00377-8.

17. Fernandez-Armenta J, Soto-Iglesias D, Silva E, Penela D, Jáuregui B, Linhart M, et al. Safety and outcomes of ventricular tachycardia substrate ablation during sinus rhythm: a prospective multicenter registry. JACC Clin Electrophysiol. 2020;6(11):1435-48. https:// doi.org/10.1016/j.jacep.2020.07.028.

18. Stein K, Dalziel K, Garside R, Castelnuovo E, Round A. Association between methodological characteristics and outcome in health technology assessments which included case series. Int J Technol Assess Health Care. 2005;21(3):277-87.

Publisher's note Springer Nature remains neutral with regard to jurisdictional claims in published maps and institutional affiliations. 